\title{
Ultrafast Localization of the Optic Disc Using Dimensionality Reduction of the Search Space
}

\author{
Ahmed Essam Mahfouz and Ahmed S. Fahmy \\ Center for Informatics Science (CIS), Nile University (NU), Cairo, Egypt
}

\begin{abstract}
Optic Disc (OD) localization is an important pre-processing step that significantly simplifies subsequent segmentation of the OD and other retinal structures. Current OD localization techniques suffer from impractically-high computation times (few minutes/image). In this work, we present an ultrafast technique that requires less than a second to localize the OD. The technique is based on reducing the dimensionality of the search space by projecting the 2D image feature space onto two orthogonal ( $x-$ and $y-)$ axes. This results in two 1D signals that can be used to determine the $\mathrm{x}$ - and $\mathrm{y}$ - coordinates of the OD. Image features such as retinal vessels orientation and the OD brightness and shape are used in the current method. Four publicly-available databases, including STARE and DRIVE, were used to evaluate the proposed technique. The OD was successfully located in 330 images out of 340 images (97\%) with an average computation time of 0.65 seconds.
\end{abstract}

\section{Introduction}

The risk of visual disabilities and blindness due to retinal diseases, whether primary or secondary to other diseases such as diabetes mellitus, could be greatly minimized by early diagnosis. An ophthalmologist needs to examine a large number of retina images to diagnose each patient; therefore, there is a significant need to develop computer-assisted diagnostic (CAD) tools for retina image analysis. The first step in any retina analysis system is to localize the optic disc (OD) [1]. The detected OD location can serve as a seed for OD segmentation, locating other structures such as the fovea [2], classifying left and right eyes in fovea-centered images, and/or a landmark to compensate large translations between retina images before applying any registration algorithm [1]. Although the OD has well defined features, developing fast and robust methods to automatically locate the OD is not an easy task due to retinal pathologies that alter the appearance of the OD significantly.

Several OD localization methods are available in literature. These methods can be classified into two main categories, appearance-based methods and model-based methods [3]. Appearance-based methods identify the location of the OD as the location of the brightest round object within the retina image. These methods include techniques such as simple threshold, highest average variation, and Principle Component Analysis (PCA) [4]. Although these methods are simple, they fail to correctly localize the OD in diseased retina images where the pathologies have similar properties to the OD. 
Model-based methods depend on analyzing the retinal vessels structure. All the currently available techniques segment the retinal vessels as a starting step. These methods incorporate techniques such as geometrical models [1], template matching [5], and convergence of vasculature [6]. Although they have relatively high accuracy, they are computationally very expensive. For example, the geometrical model-based method described in [1] achieves a success rate of 97.5\% in STARE database with a computation time of 2 minutes per image. In [2], a new OD localization method based on vasculature convergence has been described. The method achieves an accuracy of 98.77\% in STARE database with a computation time of 3.5 minutes per image.

In this work, a novel ultrafast technique for OD localization is proposed. The method is based on converting the search space from a one $2 D$ space (image space) to two $1 D$ spaces (two 1D signals). The dimensionality reduction of the search space is achieved by projecting certain image features onto two perpendicular axes (horizontal and vertical), resulting in a significant reduction of computation time. Geometric and appearance features of the OD and the vasculature structure have been incorporated into the technique to correctly identify the location of the OD. Evaluation of the proposed method using four publicly available databases showed that it achieves an accuracy of $97 \%$ with an average computation time of 0.65 seconds.

\section{Theory and Methods}

\subsection{Dimensionality Reduction and Image Features}

Searching for the OD location in a 2D space (image space) renders any localization algorithm highly expensive in terms of computational time. The idea of the proposed method is to significantly enhance the speed of the algorithm by converting the 2D localization problem into two 1D localization problems, i.e. search space dimensionality reduction. This reduction is achieved by projecting certain features of the retina image onto two orthogonal axes (horizontal and vertical). The resulting two 1D signals are then used to determine the horizontal and vertical coordinates of the OD location. The key factor needed for the success of the dimensionality reduction step is to determine the set of features that, when projected on either axes, produce a meaningful signal that can be used to localize the OD. A possible meaningful horizontal/vertical signal is a signal whose maximum value location indicates the horizontal/vertical location of the OD.

In this work, two features are selected to create the two 1D projection signals. The first, and most fundamental, feature is based on the simple observation that the central retinal artery and vein emerge from the OD mainly in the vertical direction and then branch into two main horizontal branches, see fig. 1(a). This retinal vascular structure would suggest that a vertical window (with height equal to image height and a proper width) would always be dominated by vertical edges (vertical vessels) when centered at the OD, location 1 in fig. 1(a). Although the window may contain vertical edges at other locations, e.g. small vascular branches and lesions at location 2 in fig. 1(a), it will always be populated by strong horizontal edges, i.e. the edges of the two main horizontal branches of the retinal vessels. Therefore, the integration of the difference between the vertical and horizontal edges, over a region represented by this window, can be taken as a scoring index of the horizontal location of the OD. The directionality of the retinal vessels is described by the direction of their corresponding edges in the vertical 
and horizontal edge maps of the retina image. The simple gradient operator $\left[\begin{array}{lll}1 & 0 & -1\end{array}\right]$ and its transpose are used to produce the vertical and horizontal edge maps of the retina image, respectively.

The second feature that is used in this work is based on the fact that the OD is usually a bright region. That is, the projection of the intensity values inside the window (which has height and width equal to the OD diameter) has a maximum value at the location of the OD. The following two sections will show the details of projecting these features to reduce the dimensionality of the localization problem.

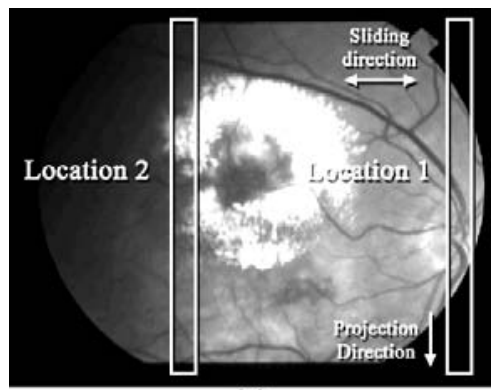

(a)

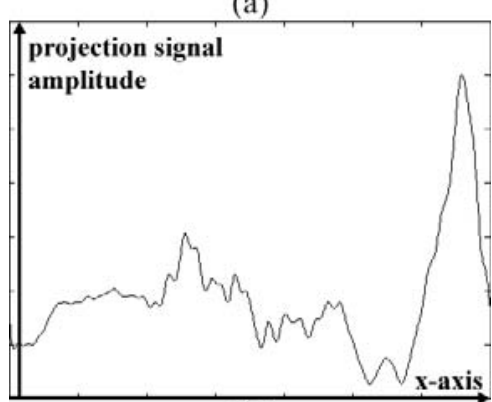

(b)

Fig. 1. (a) A retina image, from STARE database, showing the sliding window at two different locations, sliding direction and projection direction. (b) Plot of the 1D signal resulting from projecting the image features onto the horizontal axis $\left(H_{\text {projection }}\right)$.

\subsection{Horizontal Localization of the OD}

Consider a sliding window whose width and height are equal to double the thickness of a main retinal vessel and the image height, respectively. Let this window scan a retinal image from left to right and project the image features within this window onto a horizontal axis to form a 1D signal. For simplicity, assume that the only image features of interest are the image's horizontal and vertical edges. Fig. 1(a) shows an example of a retina image with the sliding window placed at two different locations $(1 \& 2)$. When the window is located over the OD (location 1), it encloses a large number of vertical edges and almost no horizontal edges. Also at location 1, the projection of pixels' intensity within the window returns a minimum value, i.e. the window contains a large number of vessels represented by low intensity pixels. At any other location in the 
image (location 2), the window may enclose a large number of vertical edges, but it will always contain a large number of horizontal edges (representing the main horizontal branches of the retinal vessels).

Fig. 1(b) shows the 1D signal resulting from projecting the two features described above on the horizontal axis. Notice that the horizontal location of the optic disc is easily identified as the location of the maximum peak of the 1D signal.

\subsection{Vertical Localization of the OD}

To determine the vertical location of the OD, the image features are projected onto a vertical axis. A vertically sliding window, centered at the pre-determined horizontal location, is defined to scan the image from top to bottom. The height and width of the window are equal to the diameter of the OD. Fig. 2(a) shows an example of a retinal image with the sliding window centered at the pre-determined horizontal location. When the sliding window is located over the OD, it encloses a large number of both vertical and horizontal edges. Also over the OD, the projection of pixels' intensity within the window returns a maximum value (the window contains a maximum number of bright pixels). At any other location along the vertical line defining the predetermined horizontal location of the OD, the window encloses fewer edges and less bright pixels. Fig. 2(b) shows the 1D signal resulting from projecting the features described above on the vertical axis. Notice that the vertical location of the optic disc is easily identified as the location of the maximum peak of the $1 \mathrm{D}$ signal.

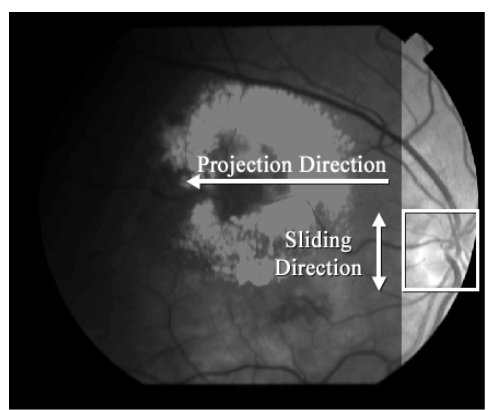

(a)

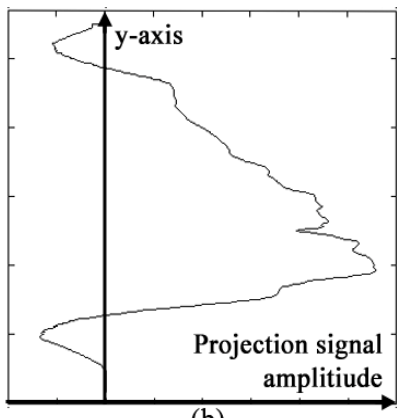

(b)

Fig. 2. (a) A retina image, from STARE database, showing the vertically sliding window, sliding direction and projection direction. (b) Plot of the 1D signal resulting from projecting the image features onto the vertical axis $\left(V_{\text {projection }}\right)$.

It is worth noting that the areas outside the camera aperture (circular region) are excluded using a binary mask generated by thresholding the red component of the image based on the method described in [7].

\subsection{Algorithm}

\section{STEP 1: Get image features}

1. Get an image of horizontal edges $\left(E_{H}\right)$ and an image of vertical edges $\left(E_{V}\right)$

2. Calculate: EdgeDiff $=\left|E_{V}\right|-\left|E_{H}\right|$; where $|$.$| is the absolute operator$

3. Calculate: EdgeSum $=\left|E_{H}\right|+\left|E_{V}\right|$ 


\section{STEP 2: Projection on the horizontal axis}

1. Define $W_{H R Z}$ as a rectangular window of size (image height, $2 \times$ main vessel width) and centered at a horizontal location $x$.

2. Slide the window $W_{H R Z}$ over the image from left to right and for each $x$,

- Calculate: $F_{H R Z}=$ sum of EdgeDiff inside the window

- Calculate: $G_{H R Z}=$ sum of pixels' intensities inside the window

- Calculate: the ratio $H_{\text {projection }}(x)=F_{H R Z} / G_{H R Z}$

3. The horizontal location of the optic disc (cand_H) is the location of the maximum value in $H_{\text {projection }}$

\section{STEP 3: Projection on the vertical axis}

1. Define $W_{V E R}$ as a rectangular window of size (OD diameter, OD diameter) and centered cand_H.

2. Slide the window $W_{V E R}$ over the image from top to bottom and for each vertical location $y$,

- Calculate: $F_{V E R}=$ sum of EdgeSum inside the window

- Calculate: $G_{V E R}=$ sum of pixels' intensity inside the window

- Calculate the value $V_{\text {projection }}=F_{V E R} \times G_{V E R}$

3. The vertical location of the optic disc $\left(c a n d_{-} V\right)$ is the location of the maximum value in $V_{\text {projection }}$

\subsection{Improving the Technique Robustness}

In some cases, the 1D signal resulting from projecting the image features on the horizontal axis $\left(H_{\text {projection }}\right)$ has multiple peaks that are close in their values (not a single prominent peak). These peaks result from various situations: (1) when the small vertical branches of the vessels are represented by strong edges and, at the same time, the OD region in the image is blurred, (2) when the image contains some artifacts. In order to improve the robustness of the proposed technique, a candidate list containing the locations of the two maximum peaks (cand_H1 \& cand_H2) in $H_{\text {projection }}$ is constructed. At each horizontal location, the vertical location is determined as described in section 2.3, which results in two possible candidate locations of the OD.

To determine which one is the correct location of the OD, the geometric properties of the bright regions around these candidates are examined. A basic assumption in this process is that if we center a vertical window at the true location of the OD, it would contain a compact bright region with eccentricity close to unity. The eccentricity is defined as the ratio of the object's major axis length to the object's minor axis length [8] and is used to measure the object's roundness, with the eccentricity of a circle equaling one. The vertical window's size is not critical and can be assumed a rectangle of dimensions: image height $\times$ OD diameter. A scoring index is defined for each candidate and is weighed by the eccentricity as follows. The brightest $3.5 \%$ pixels within the window are selected. If there is a bright object at the candidate OD location, the eccentricity of this object is calculated and the scoring index of this location, equal to $H_{\text {projection }}\left(c a n d \_H 1\right)$, is multiplied by the eccentricity of this object. If there is no object present at the candidate location, the eccentricity is set to be 0.1 and the scoring index of this location is multiplied by this eccentricity. 


\section{Results}

Four publicly available databases were used to evaluate the accuracy and the computation time of the proposed technique. The four databases are: (1) STARE database $(605 \times 700$ pixels) [9], (2) DRIVE database $(565 \times 584$ pixels) [10], (3) Standard Diabetic Retinopathy Database 'Calibration Level 0' (DIARETDB0) $(1500 \times 1152$ pixels) [11] and (4) Standard Diabetic Retinopathy Database 'Calibration Level 1' $($ DIARETDB1) $(1500 \times 1152$ pixels $)$ [11]. Accuracy and computation time results of evaluating the proposed method using these databases are summarized in Table 1. Number of images in each database is also included.

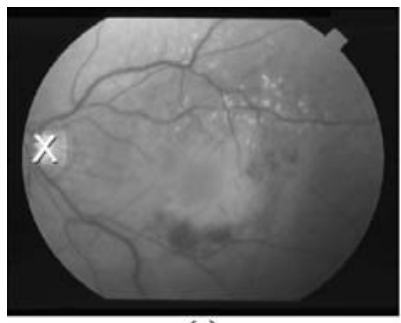

(a)

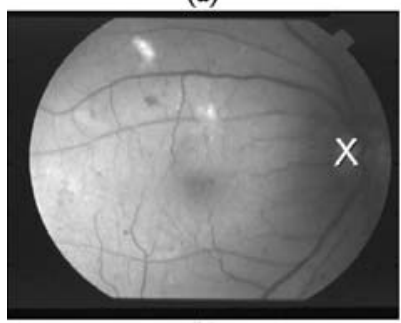

(b)

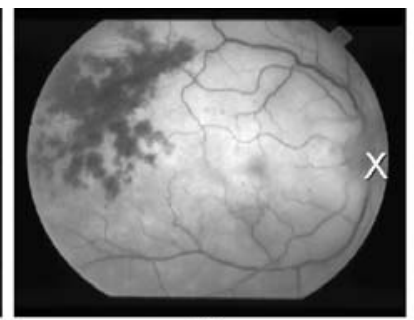

(c)

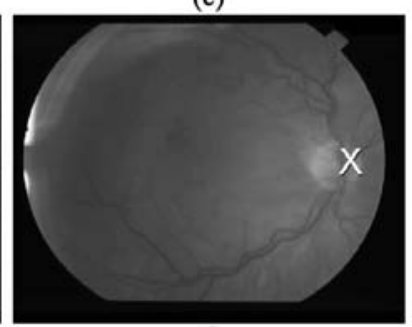

(d)
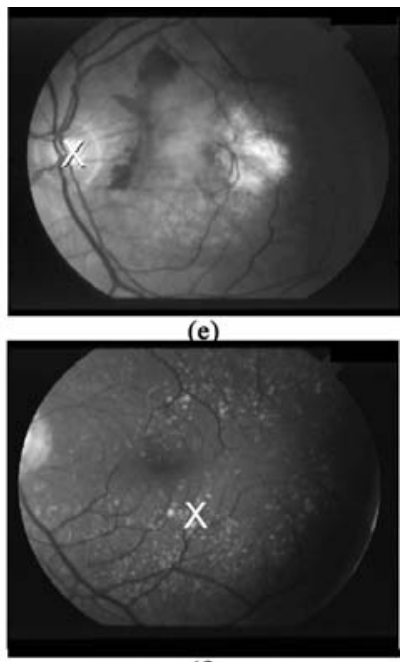

(f)

Fig. 3. Success and failure cases in 6 images selected from Stare database. (a) - (e) show successful OD localization samples. (f) shows a sample of failure in OD localization. The white ' $\mathrm{X}$ ' indicates the location of the OD as detected by the proposed method.

The detected location of the OD is considered correct if it falls within 60 pixels of a manually identified OD center, as proposed by A. Hoover et al. in [6], M. Foracchia et al. in [1] and A. Youssif et al. in [2]. The center of the OD is manually identified as the point from which all the retinal vessels emerge.

The proposed method achieved a total accuracy of $97 \%$ when tested using the four databases, i.e. the OD was correctly located in 330 images out of the 340 images tested. The OD was correctly located in 75 images out of STARE's 81 images $(92.6 \%)$ in 0.46 seconds per image with an average error of 14 pixels and a standard deviation (STD) of 15 pixels. In addition, the OD was correctly located in all the 40 images of DRIVE (100\%) taking an average of 0.32 seconds per image with an average error of 11 pixels and a STD of 11 pixels. 
Table 1. Accuracy and computation time of the proposed OD localization technique

\begin{tabular}{|c|c|c|c|c|c|}
\hline Database & $(\mathbf{1})$ & $(2)$ & $(3)$ & $(4)$ & Total \\
\hline Number of Images & 81 & 40 & 130 & 89 & 340 \\
\hline Success & 75 & 40 & 128 & 87 & 330 \\
\hline Accuracy & $92.6 \%$ & $100 \%$ & $98.5 \%$ & $97.8 \%$ & $97 \%$ \\
\hline Computation Time & $\mathbf{0 . 4 6}$ sec. & $\mathbf{0 . 3 2}$ sec. & $\mathbf{0 . 9 8}$ sec. & $\mathbf{0 . 9 8}$ sec. & - \\
\hline
\end{tabular}

\section{Discussion}

Fig. 3(a)-(e) show samples of successful localization results when applying the proposed method to selected images from STARE database. The technique of vasculature convergence [6] failed to locate the OD correctly in the first three images, while the method in [2] failed to correctly locate the OD in the $4^{\text {th }}$ image. The OD was correctly located in the $4^{\text {th }}$ image by selecting the $2^{\text {nd }}$ peak instead of the first one using the method described in section 2.5. The geometrical model-based technique [1] failed to locate the OD in the $5^{\text {th }}$ image. Fig 3(f) shows an example of an image in which the proposed method failed to locate the OD because it is partially hidden. Both methods described in $[1,6]$ also failed to locate the OD in this image.

The proposed method reduces the dimensionality of the search space from a $2 \mathrm{D}$ space (image space) of order $n \times m$ to two 1D spaces (two 1D signals) of order $n+m$, with $n$ and $m$ being the image dimensions. This dimensionality reduction is achieved through the projection of certain image features onto two orthogonal axes (horizontal and vertical). Two features are selected. The first one is the structure of the retinal vessels. The second feature is the intensity profile of the OD.

In order to increase the accuracy of the proposed method, two candidate locations of the OD are identified and additional scoring of these candidates is done by incorporating the OD geometry (represented by the eccentricity) into the technique. By investigating these two candidate locations instead of one location only, the total accuracy of the technique increased from $93.8 \%$ to $97 \%$. As shown in fig. 3, even in the presence of retinal pathologies and/or image artifacts, the selected features were unique to the OD and thus allowed proper localization with relatively high accuracy.

The most computationally demanding operation is convoluting the image with the $3 \times 1$ gradient mask used to get the edges. This operation is negligible if compared to the initial step of extracting the retinal vessels, which is required in all model-based techniques. The latter is usually achieved by applying a 2D matched filter (typically $10 \times 15$ mask) with several orientations (typically at 12 different angles) [12].

\section{Conclusion}

A new method for OD localization in retinal fundus images is presented. The method is based on reducing the dimensionality of the search space; that is, decomposing the 
2D problem into two $1 \mathrm{D}$ problems by projecting certain image features onto two perpendicular axes. The proposed method achieves accurate results in a significantly short computation time relative to currently available techniques.

\section{References}

1. Foracchia, M., Grisan, E., Ruggeri, A.: Detection of Optic Disc in Retinal Images by Means of a Geometrical Model of Vessel Structure. IEEE Transaction on Medical Imaging 23(10), 1189-1195 (2004)

2. Youssif, A., Ghalwash, A., Ghoneim, A.: Optic Disc Detection from Normalized digital Fundus Images by means of a Vessels' Direction Matched Filter. IEEE Transaction on Medical Imaging 27(1), 11-18 (2008)

3. Joshi, G.D., Gondle, V., Sivaswamy, J.: Optic Disc Detection Using Topographical Features. In: Proceedings of International EURASIP conf. (BIOSIGNAL), Funchal, Madeira Portugal (2008)

4. Li, H., Chutatape, O.: Automatic Feature Extraction in Color Retinal Images by a Model Based Approach. IEEE Transaction on Biomedical Engineering 51(2), 246-254 (2004)

5. Lalonde, M., Beaulieu, M., Gagnon, L.: Fast and robust optic disk detection using pyramidal decomposition and Hausdorff-based template matching. IEEE Transaction on Medical Imaging 20(11), 1193-1200 (2001)

6. Hoover, A., Goldbaum, M.: Locating the Optic Nerve in a Retinal Image Using the Fuzzy Convergence of the Blood Vessels. IEEE Transaction on Medical Imaging 22(8), 951-958 (2003)

7. ter Haar, F.: Automatic localization of the optic disc in digital color images of the human retina. M.S. thesis, Utrecht University, Utrecht, The Netherlands (2005)

8. Sonka, M., Hlavac, V., Boyle, R.: Image Processing, Analysis, and Machine Vision. Thomson Learning, USA (2008)

9. STARE project Website Clemson Univ., Clemson, SC, http://www.ces.clemson. edu/ ahoover/stare

10. Staal, J.J., Abramoff, M.D., Niemeijer, M., Viergever, M.A., van Ginneken, B.: Ridge based vessel segmentation in color images of the retina. IEEE Transactions on Medical Imaging 23, 501-509 (2004)

11. Kauppi, T., Kalesnykiene, V., Kamarainen, J.K., Lensu, L., Sorri, I., Uusitalo, H., Kälviäinen, H., Pietilä, J.: DIARETDB: Evaluation Database and Methodology for Diabetic Retinopathy, Technical Report

12. Chaudhuri, S., Chatterjee, S., Katz, N., Nelson, M., Goldbaum, M.: Detection of blood vessels in retinal images using two-dimensional matched filters. IEEE Transaction on Medical Imaging 8(3), 263-269 (1989) 